\title{
ENHANCE-II: An Abridged Intervention to Promote Subjective Well-Being
}

\author{
Chris C. Martin ${ }^{1}$ (D)
}

Accepted: 18 July 2021 /Published online: 13 August 2021

(c) The Author(s), under exclusive licence to Springer Nature Switzerland AG 2021

\begin{abstract}
The Enduring Happiness and Continued Self-Enhancement (ENHANCE) program, a 12-week intervention, effectively enhances subjective wellbeing and reduces negative symptoms. The current study tested an abbreviated 7-week version, ENHANCE-II, that may better fit the needs and schedules of some people. In a longitudinal study, participants $(n=51)$ took part in the self-study program and completed psychological assessments at baseline, posttest, and follow up (5 weeks). Multilevel models were used to analyze the data, with treatment group data from ENHANCE treated as the comparison. Analyses showed improvements in all four outcomes: life satisfaction (statistically significant), positive affect, negative affect, and depression. These effects were about half as strong as those in ENHANCE, but this effect reduction was partially attributable to low adherence. Effects were much stronger among participants who adhered to the program, especially for negative symptoms. Although there were no assessments at later intervals, the study suggests that ENHANCE-II intervention is likely beneficial for participants who need brief programs.
\end{abstract}

Keywords Subjective well-being · Life satisfaction · Intervention · Happiness · Positive psychology

\section{ENHANCE-II: An Abridged Intervention to Promote Subjective Well-Being}

Prior to the fourteenth century, each piece of dance music in European music stood on its own. From the fifteenth century onward, composers began to arrange a variety of dances in meaningful sequences, birthing the musical suite, which arguably reached its apogee in the works of J. S. Bach. Similarly, the first generation of

Chris C. Martin

cmartin36@gatech.edu

1 Coulter Department of Biomedical Engineering, Georgia Institute of Technology, 313 Ferst Dr., Atlanta, GA 30332, USA 
positive psychologists sought to develop interventions that stand on their own. The second generation has been investigating how to sequentially arrange, package, and customize those interventions in ways that work in the field. These suites have been called multi-component positive interventions (Hendriks et al., 2020).

In both the first and second generations, the proponents of positive psychology have worked on translating basic science into interventions that increase happiness, which is also termed subjective well-being. It is a truism that people want their children to be happy, and view their happiness as an ultimate end (Taaffe Young et al., 1998; Wittenberg et al., 2017). The empirical research on happiness also suggests that it causes better life outcomes (Lyubomirsky, et al., 2005a, 2005b). For instance, happiness seems to cause better health and longevity (Diener et al., 2017) and better job performance (Peterson et al., 2011). Happiness also prospectively predicts income (De Neve \& Oswald, 2012). The causal direction in some studies can be ambiguous (Rohrer \& Lucas, 2020) but the available evidence suggests that happiness is an antecedent rather than a consequence of these outcomes (e.g., De Neve \& Oswald, 2012). For these reasons, the discovery of practices that promote happiness have been especially popular both inside and outside academia.

What daily practices and habits can change happiness? Psychological research suggests that people become happier when they engage in practices such as maintaining a gratitude journal (but not updating it too often), valuing time over money, engaging in more social activity, and learning cognitive behavioral therapy (Algoe et al., 2013; Fava, 1999; Lyubomirsky, et al., 2005a, 2005b; Sandstrom \& Dunn, 2014a). Can this change be sustained? Research indicates that two principles of hedonic adaptation prevention, variety and appreciation, can be leveraged to sustain one's gains (Armenta et al., 2014). People can learn to apply these principles through positive psychology interventions. In recent years, practices from both streams of research-basic practices and sustainability-have been packaged into multi-component interventions, which are more comprehensive than the singlecomponent interventions from the initial era of positive psychology (A. C. Parks \& Schueller, 2014). A particular feature of these interventions is the combination of exercises that target hedonic and eudaimonic well-being components into an integrated program (Hendriks et al., 2020). The hedonic components of the intervention typically teach people how to extract enjoyment from daily life. The eudaimonic components enhance a person's ability to align their goals and activities with their character strengths and intrinsic motives.

The efficacy of multi-component programs suggests they have small to moderate effects on life satisfaction (Hedges' $g=0.34)$, psychological well-being $(g=0.39)$, depression $(g=0.29)$, and anxiety and stress ( $g=0.35$; Hendriks et al., 2020) where "small" and "moderate" refer to typical intervention effects (Lipsey \& Wilson, 1993). Although a small effect may seem unimpressive, it can be subjectively significant from the participant's point of view. Moreover, large effects may be unattainable given the stability of happiness. Some multi-component interventions target clinical populations, while others focus on the general public (Hendriks et al., 2020). Among both types, there have been many low-quality intervention studies, so in recent years, one focus has been the use of better methods, such as randomized controlled trials. Low-quality studies have inflated effect sizes, which can exaggerate the 
true effects (Hendriks et al., 2020). One recent multi-component intervention is the Enduring Happiness and Continued Self-Enhancement (ENHANCE) the program, and the current study is a conceptual replication of the first ENHANCE trial.

\subsection{ENHANCE Programs}

The ENHANCE program is a 12-week lesson and exercise plan for sustainably increasing well-being (Heintzelman et al., 2020). The investigators in ENHANCE-I sought to create a program for a non-clinical population and test it using a randomized clinical method (Heintzelman \& Kushlev, 2020). The method and predictions were preregistered in an article (Kushlev et al., 2017) and a repository (https:// clinicaltrials.gov/ct2/show/NCT02782611). The duration of the program was set to 12 weeks because meta-analytic findings suggest that duration predicts efficacy (Sin \& Lyubomirsky, 2009). The program was composed of four units: introductory material, the core self, the experiential self, and the social self. A detailed review of the mechanisms of change can be found in the preregistration.

The section on the core self teaches participants to recognize their values, goals, and character strengths. In the values chapter, participants affirm their core values, which maximizes well-being by reminding people of their psychological immune system and by triggering positive feedback processes (Howell, 2017). The goals chapter involves a clarification of intrinsic motives, which helps participants set goals that engender more satisfaction (Deci \& Ryan, 1985). The strengths chapter aims to help participants recognize and deploy their strengths, a practice that can improve engagement and flow (Buschor et al., 2013; Forest et al., 2012).

The section on the experiential self teaches participants to extract satisfaction and inhibit distress in daily life. The mindfulness chapter introduces participants to exercises that help them to guided mindfulness practices, which help people with emotional regulation and forms of interpersonal distress (Khoury et al., 2015). The chapter on dealing with negativity trains participants to use self-compassion, a recently developed technique, and cognitive restructuring, a technique that draws on second-wave cognitive behavioral therapy. Self-compassion buffers against anxiety and self-criticism (Neff et al., 2007), and cognitive restructuring allows participants to recognize mental events in which irrational thought patterns cause distress (Butler et al., 2006).

The module on the social self teaches participants to bolster their current relationships and begin new relationships. Building and maintaining social ties can fulfill relatedness needs (Gottman \& Levenson, 1992; Sandstrom \& Dunn, 2014b). It also includes a section on kindness and altruism, which can fulfill relatedness, competence, and autonomy needs (Dunn et al., 2014).

ENHANCE-I was evaluated in a randomized controlled trial with both in-person and online versions (Heintzelman et al., 2020). The in-person version was tested on 54 community adults in Canada, and the online version on 100 community adults in the U.S. Participants were sorted into gender-balanced experimental and control groups. The mode of administration had no discernible effect on effectiveness. Conditional growth models showed that ENHANCE-I was effective in improving life 
satisfaction at both posttest and three-month follow-up. An improvement in depression symptoms was also detected, but the effect size was smaller and non-significant. This improvement was also sustained through the follow-up. There were also improvements in positive affect and negative affect at posttest, but these outcomes regressed toward the pre-study baseline from posttest through follow-up. A fourth assessment showed a maintenance of gains over six months in the original online treatment condition $(n=39)$, and the waitlist group also benefited after they received the treatment. A limitation of the study was that participants were aware of the study's goal, and the recruited participants represented the set of community members who were engaged in the pursuit of happiness.

\subsection{The Current Study}

The current study tests the efficacy of ENHANCE-II, an abridged version of ENHANCE designed to fit the schedules of busy people. In the current study, the sample was comprised of students at a highly selective engineering college in the U.S. These students cope with time pressure and might not adhere to longer programs. Person-activity fit can determine the efficacy of positive psychology interventions (Lyubomirsky \& Layous, 2013; Proyer et al., 2015), and, on one hand, shorter programs may be a better fit for busy people. On the other hand, the smaller dosage may reduce efficacy because stronger dosages are typically more beneficial (Lyubomirsky \& Layous, 2013) and longer interventions are more effective on average (Sin \& Lyubomirsky, 2009). However, the dose-response association can violate our intuitions - in gratitude interventions, moderate doses are effective, whereas high doses are ineffective (Lyubomirsky, et al., 2005a, 2005b). Although little is known about why duration matters, longer programs may cause the formation of good habits, and the internal variety may also help participants find activities that fit them, whereas an abridged intervention may inhibit the ability of participants to fully understand and apply concepts. However, shorter interventions may be more effective for people with tighter schedules because program completion is more likely. They may also more engaging for people with high cognitive ability because the compression of material is less likely to induce boredom due to repetition. A short intervention may therefore be suitable for undergraduate students at competitive colleges, who comprise the sample in the current study. Their workload and stress increase toward the middle of each 15-week semester (Baghurst \& Kelley, 2014; Pitt et al., 2018; Stiwne \& Jungert, 2010), and they may not adhere to longer programs.

The effectiveness of ENHANCE-II was measured by comparing ENHANCEII results with ENHANCE-I results. Given evidence that ENHANCE-I is effective-and the prior evidence that its component activities are effective-I did not recruit participants for a control condition, but instead focused on relative effectiveness. Participants filled measures of well-being at three timepoints: pre-intervention, post-intervention, and follow up. The primary measures used in ENHANCE-I were also used in this study, making the ENHANCE-I data usable for comparisons. Unlike ENHANCE-I, the current study did not add assess domain satisfaction, 
mode-of-delivery effects, targeted skill development, and peer-reported subjective well-being because it was funded by a small grant and there was only one investigator, which necessitated a smaller workload. Nevertheless, it is likely that the mechanisms of change are similar across both the unabridged and abridged version due to content overlap.

\section{Method}

\subsection{Participants}

Participants were 51 students at the Georgia Institute of Technology, a highly competitive university. It is ranked 38th among world universities by Times Higher Education, and its undergraduate engineering program is ranked 4th in the nation by U.S. News and World Report (Times Higher Education, 2021; U.S. News \& World Report, 2021). Participants were in the age range of 18-25. The gender distribution was $62.8 \%$ female, $35.3 \%$ male, and $2 \%$ unknown. The racial/ethnic distribution was $33.3 \%$ non-Hispanic White, 4\% Hispanic White (or Hispanic only), 13.7\% Black or African-American, 43.1\% Asian or Asian-American, 2\% Middle Eastern, Arab, or Arab-American, and 4\% other or unknown. In all, $60.8 \%$ were 18 years old, $15.7 \%$ were 19 , and the remainder were in the 20-25 range or had age missing; $78.4 \%$ were single and $19.6 \%$ were in a relationship; and $78.4 \%$ were heterosexual, $3.9 \%$ were gay/lesbian, and $11.8 \%$ were bisexual. Eighty percent of participants self-identified as domestic students and $17.7 \%$ as international students. Approximately $74 \%$ percent of participants were first-year students. The social class of most participants was upper class: $77.5 \%$ reported that their father (or paternal equivalent) and $62.5 \%$ reported that their mother (or maternal equivalent) had a bachelor's degree or higher.

Of the 51 participants, 49 completed the first wave, 38 completed the second wave, and 41 completed the third wave. Thus, 38 participants completed all three waves. Tests showed that there were no significant differences in life satisfaction $(p=0.89)$, positive affect $(p=0.78)$, negative affect $(p=0.93)$, and depression $(p=0.73)$ at wave 1 between those who filled all three waves and those who did not.

\subsection{Procedure}

Recruitment for the study was conducted during the spring and summer of 2020. The study was conducted in the fall of 2020. Study procedures were deemed exempt from review by an Institutional Review Board at Georgia Institute of Technology. The methodology was a simplified version of the method in the ENHANCE-I study by Heintzelman et al. (2020).

A G*Power analysis for repeated measures ANOVA was used to estimate power (Faul et al., 2007). A repeated-measures ANOVA resembles a multilevel model with waves nested under persons, used for the actual analysis. The power analysis estimated $95 \%$ power to detect an effect size $(f)$ of 0.25 with a sample size of 43 . This estimate was produced by modeling a repeated-measures ANOVA with a correlation 
of 0.5 among repeated measures and no correction for non-sphericity. The recruitment target was set to 50 participants, and grant funding was obtained to compensate this sample. A post hoc analysis indicated that the study had $85 \%$ power to detect the most problematic effect from baseline to follow-up (repeated correlation $=0.50$, Greenhouse-Geisser epsilon $=0.69$ ) given that 40 participants filled waves 1 and 3.

Supplementary documents and data are available on OSF at https://osf.io/4tv5n/.

\subsubsection{Workbook Editing}

Using the ENHANCE-I workbook as a basis, a significantly shorter workbook was created for participants to independently go through the program (downloadable at https://osf.io/4tv5n). This 57-page workbook was derived from the 230-page package of printed material used in ENHANCE-I; the word count was cut from 26,400 to 13,500. A 43-page Companion book exclusively containing exercises was also used in ENHANCE-I, but not ENHANCE-II. A side-by-side report and an interleaved report of differences between the two workbook is available on the OSF page.

Figure 1shows how ENHANCE-I content was abridged, excised, and rearranged to fit a seven-week schedule. The first major change was that gratitude and savoring chapters were excised. The available evidence, which consists of two studies, indicates that gratitude exercises have no discernible effects in India, Taiwan, and South Korea, where such exercises stir up feelings of indebtedness (Layous et al., 2013; Shin et al., 2020). Due to the demographic composition at the targeted institution, I predicted-accurately - that many participants would be Asian or second-generation Asian Americans. On average, second-generation immigrants share the values of their parents (Polavieja, 2015). This would make the gratitude module problematic. In addition, participants in the trial of ENHANCE-II were enrolled at an engineering college, where people valorize a culture of rigor and overwork (Campbell et al., 2018; Stiwne \& Jungert, 2010). They may have felt resentful if prompted to think about gratitude and appreciation, and these modules were therefore better suited for removal. Ultimately, the removal of these two modules was a subjective decision, based on weak evidence. However, some content removal was necessitated by the goal of condensing the workbook.

The second major change was condensation. To abridge the material, several paragraphs in each chapter were deleted or shortened. Paragraphs were deleted if they elaborated on content that had already been presented. Most of the writing exercises were retained to make the book more appealing than a typical undergraduate textbook. Educational exercises that require activity rather than reading on the user's part are also more effective (Chi \& Wylie, 2014). Explain-to-a-friend writing exercises were added to add variety to some chapters, a revision based on empirical findings about the efficacy of writing (King, 2001). To compensate, I reduced the number of blanks in some fill-in-the-blank exercises. I also substituted simple words for technical words and used Lanham's (1992) paramedic method to clarify the prose.

The third major change was the compression of end-of-chapter activities. In ENHANCE-I, each module ended with three activities: Activity of the Week (for the present week), Companion Book Activity (also for the present week), and AfterThis-Week activity (for subsequent weeks). Only one or two activities from these 
sets were retained. For instance, in the social-activities module, the plan-making exercise for three social interactions was retained, and in the negative-thoughts module, an activity involving writing about negative events at three preset times was retained.

\subsubsection{Recruitment}

Recruitment was conducted in two waves. In the first wave, notices were posted via web announcements and sent by email to students in one psychology and two biomedical engineering courses. Students were invited to email the investigator, who sent them a link to the screener form. In the second wave, emails were sent to incoming first-year students participating in a voluntary orientation program; these emails were sent in blocks of 50 .

Screening was done using a shortened 8-item version (see below) of the 9-item Patient Health Questionnaire (PHQ-9; Kroenke et al., 2001), which assesses depression. In ENHANCE, the cut-score was initially set to $\leq 14$, but I raised the cut-score to $\leq 19$ in the first wave and $\leq 20$ in the second wave, after finding elevated scores in a large proportion of applicants. The elevated scores were probably due to the pandemic; research with representative samples shows that a wave of psychological distress in April 2020 was followed by a return to the baseline in June 2020 (Daly \& Robinson, 2020). Consistent with this trend, the PHQ score for participants dropped dramatically from the recruitment period $(M=13.5,95 \% \mathrm{CI}=[12.6,14.4])$ to the first wave $(M=4.0,95 \% \mathrm{CI}=[3.0,5.0]), t(46)=23.08, p<0.0001, d=-3.4$. This change suggests that many applicants were having a normal reaction to the pandemic and were not suffering from major depressive disorder (Robinson \& Daly, 2020). Furthermore, there was also no theoretical reason to expect that ENHANCE would be ineffective among people suffering from depression. The creators of ENHANCE-I excluded participants suffering from depression because the program was not advertised or intended as a treatment (Kushlev et al., 2017). It was not due to the expectation that the program would be less effective among depressed people. The inclusion of depressed people makes the sample more representative of the population. Participants with disqualifying scores were informed they had depression symptoms and they received counseling information (see Appendix).

\subsubsection{Participation Schedule}

The schedule of activities, surveys, and compensation is in Table 1. The informed consent form was sent to all 57 screened participants. Of these participants, 52 gave informed consent, after which one dropped out due to their own assessment that they would be unreliable. Three participants who consented to participate were not giving the screening form because they expressed interest in the study only a few days before the study initiation, and more participants were needed at this point. Thus, 51 participants submitted the first survey wave after giving informed consent. A smaller number of participants filled successive waves and completed the full workbook but following best practices, data from all participants were used in model estimation (Heintzelman \& Kushlev, 2020). Approximately 30 days after the study began, 
Table 1 Schedule of activities and measurement

\begin{tabular}{|c|c|c|c|}
\hline Week no. & Date & Participant activity & Measurement \\
\hline 0 & Aug. 16-23 & Put program schedule in personal calendar & Wave $1(n=51)$ \\
\hline 1 & Aug. 23-29 & $\begin{array}{l}\text { Read Introduction and Chapter 1; do related } \\
\text { activities }\end{array}$ & \\
\hline 2 & Aug. 30-5 & Read Chapter 2; do related activities & \\
\hline 3 & Sep. 6-12 & Read Chapter 3; do related activities & \\
\hline 4 & Sep. 13-19 & Read Chapter 4; do related activities & \\
\hline 5 & Sep. $20-26$ & Read Chapter 5; do related activities & Progress update $(n=50)$ \\
\hline 6 & Sep 27-Oct. 3 & Read Chapter 6; do related activities & \\
\hline 7 & Oct. $4-10$ & $\begin{array}{l}\text { Read Chapter } 7 \text { (Conclusion); do related activi- } \\
\text { ties }\end{array}$ & \\
\hline 8 & Oct. $11-18$ & No Activity $^{\mathrm{a}}$ & \\
\hline 9 & Oct. 19-26 & & Wave $2(n=38)$ \\
\hline $10-13$ & Oct. 27-Nov. 22 & No Activity & \\
\hline 14 & Nov. $23-30$ & & Wave $3(n=41)$ \\
\hline 15 & Dec. $1-8$ & Receive $\$ 30$ gift card if 3 waves completed & \\
\hline
\end{tabular}

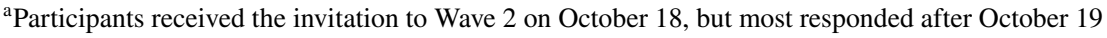

one participant emailed the investigator expressing an intention to drop out due to a bereavement and "having a rough time this entire semester." The reason for the remaining attrition is unknown.

Participants were compensated with a \$30 Amazon gift card after Wave 3 if they submitted Waves 1,2, and 3. The decision to provide compensation at the end was to reduce extrinsic motivation for program completion (Deci et al., 1999).

\subsubsection{Workbook Delivery and Completion}

The initial plan was to give each participant a printed workbook, but due to pandemic-related contingencies, the plan was revised: students were either given a printed version or an electronic Word file based on their preference. Unlike in the ENHANCE-I study, there was no maintenance phase in which participants received reminders to continue practicing their activities because there was a much shorter gap between conclusion and follow-up timepoints.

The week- 5 progress report was filled by 50 participants, including three participants who skipped Waves 1-3. Respondents were asked what proportion of the workbook they had completed, and four chose "about 1\%," two chose "about 25\%," 10 chose "about 50\%" 26 chose "about 75\%" and eight chose "about 100\%." In Wave 3 , participants were asked which chapters of the workbook they had completed; of the 41 respondents, these are the number who completed each respective chapter: $34,33,35,32,30,25$, and 18. Three participants skipped all the chapters. This attrition aligns with the claim that longer interventions may be a poor fit for college students. The final progress report, included in the Wave 3 survey, showed that most 
non-adhering participants gave up after using a set of consecutive chapters, although four participants completed a set of non-consecutive chapters.

\subsection{Measures}

Four primary measures used in the ENHANCE study were used in ENHANCEII. Cronbach's alpha $(\alpha)$ and Raykov's factor reliability $(\Omega)$ were computed using Wave-1 data. The item-level reliability was computing using a three-level hierarchical linear model (Nezlek, 2017). The item-level reliability can be low due to variability in item skewness and item means, and stability at the person-level. Scale reliabilities are reported in Table 2.

\subsubsection{Life Satisfaction}

Life satisfaction was measured with the five-item Satisfaction With Life Scale (SWLS; Diener et al., 1985). In the conventional form, respondents rate statements on a five-point scale. Although I intended to use the five-point scale, I erroneously inserted seven points, ranging from 1 (strongly disagree) to 7 (strongly agree). The SWLS has been tested for reliability and sensitivity (Diener et al., 2013). An example item is "The conditions of my life are excellent." Item scores were averaged to compute the life satisfaction score.

\subsubsection{Positive and Negative Affect}

The Scale of Positive and Negative Experience (SPANE; Diener et al., 2010) was used to measure affect. It improves upon the commonly used Positive and Negative Affect scale (Watson et al., 1988) by focusing more on emotion than arousal, and more on frequency than intensity of emotions. Frequency is more relevant to happiness than intensity (Diener et al., 1991). The SPANE was also designed to be cross-culturally valid (Silva \& Caetano, 2013; Sumi, 2014). Six items each tap into positive and negative affect in the past two weeks, and respondents rate experience frequency on a scale from 1 (very rarely or never) to 5 (very often or always) with this order of items: positive, negative, good, bad, pleasant, unpleasant, happy, sad, afraid, joyful, angry, and contented. In its original version, the scale is keyed to the

Table 2 Reliability estimates and person-level descriptive statistics and correlations $(\mathrm{N}=51)$

\begin{tabular}{lcccccccc}
\hline & $\alpha$ & $\Omega$ & ILR & $M$ & $S D$ & Life satisfaction & Positive affect & Negative affect \\
\hline Life satisfaction & .82 & .83 & .10 & 5.3 & 0.9 & & & \\
Positive affect & .90 & .91 & .69 & 3.8 & 0.7 & $.56^{* * *}$ & & \\
Negative affect & .74 & .74 & .38 & 2.1 & 0.6 & $-.48^{* * *}$ & $-.60^{* * *}$ & \\
Depression & .75 & .75 & .15 & 3.3 & 1.8 & $-.42^{* *}$ & $-.39^{* *}$ & $.50^{* * *}$ \\
\hline
\end{tabular}

Note $\mathrm{ILR}=$ item-level reliability

${ }^{* * * *} p<.001{ }^{* *} p<.01{ }^{*} p<.05$ 
last four weeks, but following ENHANCE-I, I used two weeks. Item scores were averaged to compute separate scores for positive and negative affect.

\subsubsection{Depression}

ENHANCE-I was developed to increase subjective well-being, but the authors also evaluated its effect on depression because other positive-psychology interventions have decreased depression (Sin \& Lyubomirsky, 2009). The nine-item Patient Health Questionnaire (PHQ-9; Kroenke et al., 2001) was used to measure depression in ENHANCE-I. A shortened eight-item version was used in the current study. The ninth item, which asks about "Thoughts that you would be better off dead or of hurting yourself in some way" was excluded. Because it refers to suicidal ideation, its inclusion would have required a full protocol review by the Institutional Review Board, which would have delayed recruitment indefinitely.

The PHQ-9 was developed for primary-care clinicians to make diagnoses of depression and related mental disorders, and is usable for both categorical diagnoses of depression and continuous measurement of symptom severity (Kroenke et al., 2001). Respondents are asked how often they have been bothered by specific symptoms over the last two weeks. Symptoms include "little interest or pleasure in doing things" and "feeling bad about yourself_-or that you are a failure or have let yourself or your family down." Items are rated on a scale from 0 (not at all) to 3 (nearly every day). Item scores are summed.

\subsection{Data analysis}

Data were analysis using multilevel modeling, which handles missing data better than repeated-measures ANOVA (Nezlek, 2011). All analyses were conducted by the first author. Models were fit using restricted maximum likelihood instead of maximum likelihood because of the small sample size. Dummy variables were created for the second and third waves. Persons were entered at level two and waves were entered at level 1 . The only predictors were the dummy variables for the second and third waves, and their slopes were allowed to vary at level 2. However, if the estimation indicated no slope variance, the model was retested with a fixed slope. In the results tables, a blank cell for slope variance indicates a fixed slope. A variable slope indicates that there was variability in the effectiveness of the program across participants. I did not test whether demographic factors explained such variability because of inadequate statistical power.

Because there was likely to be variation in adherence, I used a second set of models to determine whether workbook persistence moderated the effects of the intervention. I treated the number of chapters completed as a continuous variable, and entered this variable, and the product of this variable and the dummy codes for waves 2 and 3 into a model. Slopes were fixed or allowed to vary as before. 


\section{Results}

Table 2 displays the reliability of measurement scales, person-level means, withinand between-person standard deviations, and correlations at the person-level. The correlations between positive and negative aspects of well-being were in the theorized direction.

The results of the multilevel models are in Table 3. There was clear evidence of enhanced life satisfaction. Participants' life satisfaction was higher than baseline in both the posttest and five-week follow-up with no evidence of post-intervention decline. There was marginal evidence of improvement in positive affect and negative affect - the effects were in the theorized direction, but either marginally significant $(p<0.10)$ or non-significant. The effect for depression was also in the anticipated direction but non-significant. The effect sizes (Cohen's $d$ ) at posttest, displayed in bold in Table 4, were non-trivial. At posttest, they ranged in absolute magnitude from 0.24 to 0.37 ; at follow-up, they ranged from 0.16 to 0.39 . These results suggest that ENHANCE-II may have had triggered improvements across all variables at posttest, but there was only strong evidence of effectiveness for life satisfaction.

The discernible gain in life satisfaction was enduring. The marginal improvement in depression also appeared to be enduring, whereas the marginal improvement in negative affect was slightly accentuated at follow-up. Given that the within-subjects design was highly powered, these results suggest that ENHANCE-II is effective for improving life satisfaction but it may need to be modified to improve other outcomes.

\subsection{Comparison with ENHANCE-I}

Table 4 shows a comparison of ENHANCE-I and ENHANCE-II effects. A detailed version of this table is on the OSF page. The follow-up interval was three months in ENHANCE-I and five weeks in ENHANCE-II, but the follow-up contrast is reported for transparency. The effect size differences in the ultimate column show the difference across experimental groups, the focal contrast. As shown in the rightmost column under each outcome, the effect sizes at follow-up for ENHANCE-II vs. ENHANCE-I were 0.37 versus $0.67,0.31$ versus. $0.61,-0.28$ versus -0.65 , and -0.24 versus -0.63 for the respective outcomes. Thus, the ENHANCE-II effect was approximately $50 \%$ of the ENHANCE-I effect for life satisfaction and positive affect, slightly less than $50 \%$ for negative affect, and about $40 \%$ for depression at posttest. The effect-size ratio was also in the 40-50\% range at follow-up, indicating that ENHANCE-II was about as half as effective as ENHANCE-I. The secondary contrast of interest was between the ENHANCE-II sample and the ENHANCE-I control condition. In life satisfaction and negative affect, participants in ENHANCEII had greater gains than those in the ENHANCE-I control condition. At posttest, but not follow-up, there was also greater relative improvement in positive affect and depression in ENHANCE-II. 


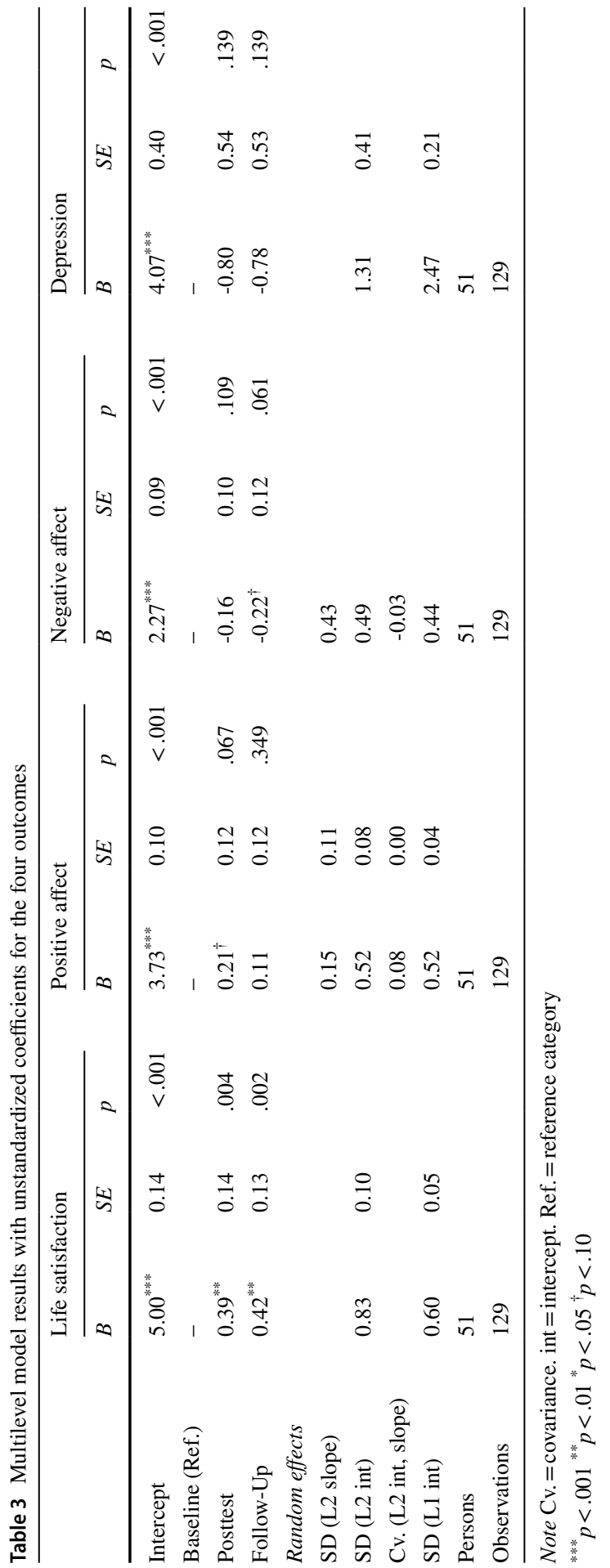


Table 4 Effect sizes for withinperson change in ENHANCE and ENHANCE-II

\begin{tabular}{lllllll}
\hline Study & Condition & $N$ & \multicolumn{4}{l}{ Effect size (Cohen's $d$ ) } \\
\cline { 3 - 7 } & & & Life Sat & PA & NA & Depression \\
\hline \multirow{2}{*}{ Posttest } & I Control & 67 & 0.29 & 0.04 & -0.12 & -0.07 \\
& II & $\mathbf{3 8}$ & $\mathbf{0 . 3 7}$ & $\mathbf{0 . 3 1}$ & $\mathbf{- 0 . 2 8}$ & $\mathbf{- 0 . 2 4}$ \\
& I Exp & 66 & 0.67 & 0.61 & -0.65 & -0.63 \\
Follow-Up & I Control & 65 & 0.29 & 0.16 & -0.19 & -0.58 \\
& II & $\mathbf{4 1}$ & $\mathbf{0 . 3 9}$ & $\mathbf{0 . 1 6}$ & $\mathbf{- 0 . 3 9}$ & $\mathbf{- 0 . 2 3}$ \\
& I Exp & 62 & 0.69 & 0.46 & -0.38 & -0.51 \\
\hline
\end{tabular}

Note ENHANCE-II effect sizes are in bold. A supplemental table shows effect sizes with all means and SDs

\subsection{Adherence as Moderator}

Because of the variance in adherence, I tested whether workbook persistence moderated the effects of the intervention. The results indicate that participants who adhered to the program were more likely to benefit from ENHANCE-II. All interaction effects were in the expected direction, and two were statistically significant as noted in Table 5. I conducted a comparison of follow-up outcomes among participants who completed one chapter versus all seven chapters by estimating marginal effects $(\mathrm{dy} / \mathrm{dx})$. These marginal effects were as follows: life satisfaction $(0.12$ vs 0.56$)$, positive affect $(-0.27$ vs. 0.29$)$, negative affect $(0.39$ vs. -0.47$)$, and depression (2.72 vs. -2.11$)$. The full (seven chapter) adherence effects for subjective well-being, negative affect, and depression were significantly different from zero, $p=0.001$. With negative affect and depression, the pairs of 95\% CIs were nonoverlapping for low versus. high adherence. Thus, adherence strongly moderated the effect of the intervention.

\subsection{Depression and Adherence}

Depressed participants could have been more likely to quit the workbook, confounding the adherence effect. However, baseline PHQ scores predicted workbook completion and showed that depressed participants were more likely to adhere to the workbook. Baseline PHQ score was positively correlated with mid-program progress $(r=0.20, p=0.18)$ and with number of chapters completed by posttest $(r=0.36, p=0.02)$. Among those who reported mid-semester progress, $100 \%$ of participants with PHQ $>2$ (27 of 47) had completed at least half the workbook, whereas $20 \%$ of those with PHQ $\leq 2$ had not. Among those who reported progress at posttest, all participants with PHQ $>4$ ( 24 of 40 ) had completed 4 or more chapters, whereas $13 \%$ of those with PHQ $\leq 4$ had not. Some participants who were already faring well may have discontinued the program, which explains these results. Unmeasured confounding and reversion to the mean cannot be ruled out. 


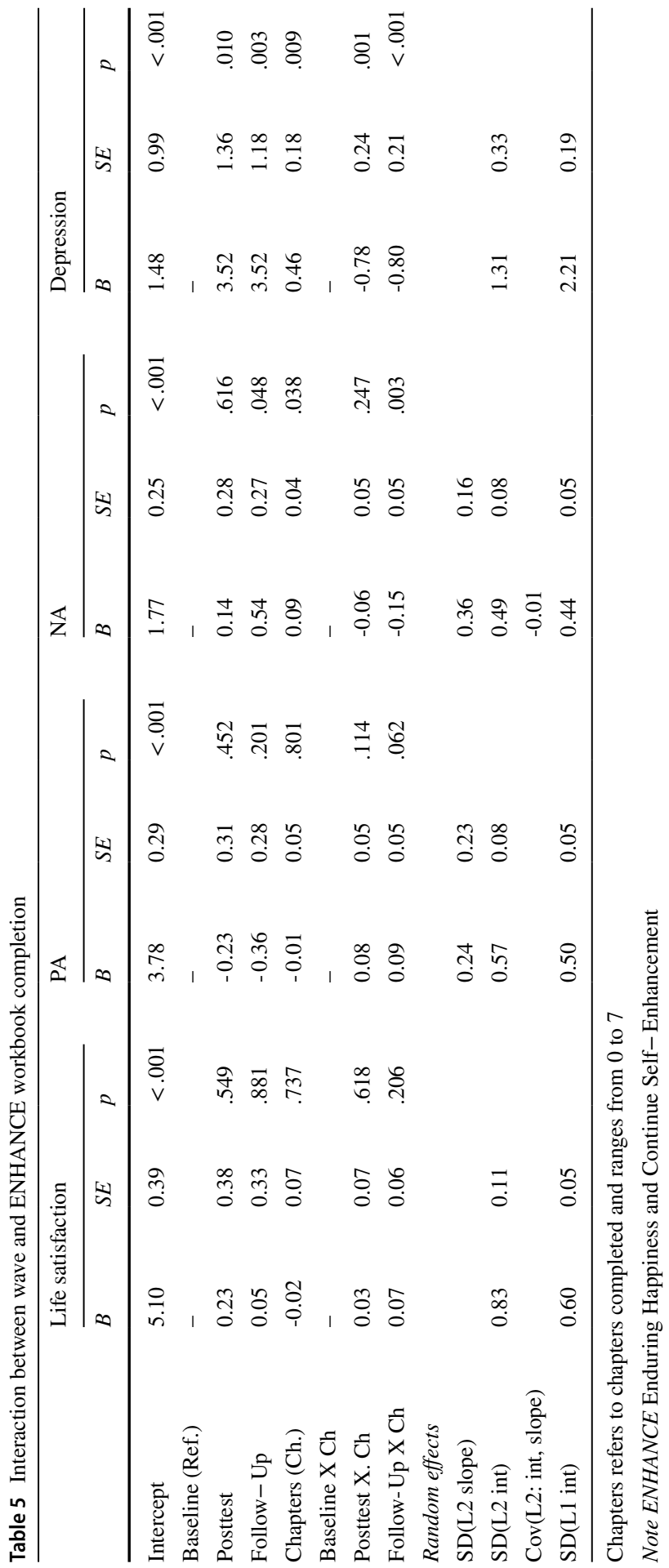




\section{ENHANCE-I ENHANCE-II}

Introduction

1. Values

0. Introduction +

Identify most important values; affirm those values

1. Seeing Your Values and Goals and engage in parallel activities

2. Goals

Pursue goals that are intrinsically motivated and approach oriented

3. Character Strengths

2. Personal Strengths

Identify one's top character strength and use those strengths intentionally

4. Mindfulness

Comprehend mindfulness; practice engaging in mindfulness and related techniques

3. Dealing with the Negative

5. Dealing with negativity

Practice self-compassion and cognitive behavioral techniques for negating irrational thoughts

\section{Savoring}

4. Mindfulness

Savor activities in the present and cherish past experiences

7. Close relations

Increase ratio of positive to negative interactions; practice social activities like sharing one's joys

\section{Gratitude}

Express gratitude in daily life and engage in gratefulness at fixed intervals

9. Social interaction

Explore unfamiliar ways to engage in casual social interactions

10. Prosocial behavior

6. Contributing to the Happiness of Others

Learn how to help and show kindness to others; learn how to be altruistic

Conclusion

7. Conclusion and Future Plan

Formulate plans for continuation of these activities

Fig. 1 Remapping of Content from ENHANCE-I to ENHANCE-II

\section{Discussion}

Previous research indicates that well-being can be sustainably elevated through a 12-week multi-component program (Heintzelman et al., 2020). The current results indicate that a short version of this program is also effective though not to the same degree. Among college students at a highly competitive university, the ENHANCEII program induced an improvement in well-being at the end of seven weeks which was partially sustained. A comparison of effect sizes across ENHANCE versions suggests that ENHANCE-I, the original intervention by Kushlev and colleagues, may be preferable. However, in a population where enrollment in a 12-week program is likely to be low due to time pressure, ENHANCE-II may be better than no intervention at all. 
The improvements across the four outcomes were not trivial. Among multicomponent interventions, the mean effect size (Cohen's $d$ ) is 0.34 for subjective well-being and 0.29 for depression at posttest $(0.27$ and 0.45 respectively at followup). When low-quality studies are dropped, this effect size drops to 0.26 for subjective well-being and 0.14 for depression at posttest (Hendriks et al., 2020). In ENHANCE-II, which would be classified as a medium-quality study, the effect sizes are close to these latter averages. However, the improvement in depression, though enduring, could have plausibly occurred with no treatment, given control data from ENHANCE, and the improvement in positive affect was transient.

One explanation for the program's efficacy is a placebo effect. Previous research has investigated the nature of placebo effects on similar interventions and found that people who select into a positive intervention activity that is deceptively given the neutral label "cognitive exercises" do not experience the same benefits as those who select a "happiness intervention" with identical content (Lyubomirsky et al., 2011). However, the same study showed that the "happiness intervention" group only benefited if they were in a positive-activity condition, not a neutral-activity condition. Had there been a strong placebo effect, all participants in the "happiness intervention" should have benefited. In any case, placebo effects in subjective well-being studies are impossible to eliminate through objective reports. Unlike quality of life or objective need satisfaction, subjective well-being arises from the perspective of the subject (Diener et al., 2018).

The results showed some variance across participants and across outcomes. Adherence to the program was imperfect, which explained variance across participants. A moderation analysis suggested that if all participants had adhered to the intervention, the aggregate effects would have been stronger. Regarding outcomes, the greater improvements in negative symptoms and smaller change in positive symptoms may reflect two factors. First, the savoring and gratitude components, which enhance positive affect, were excluded in ENHANCE-II, whereas modules pertaining to negative affect were retained. Second, the modules on negative affect and mindfulness were in the third and fourth module; participants who quit in week 2 did not benefit from them.

One apparent drawback of ENHANCE-II is that some gains seemed to be only partially sustained. However, there was some effect dissipation even in ENHANCEI. Furthermore, there were discernible effects at the follow-up for participants who adhered to the workbook. A program that ensures full adherence to the workbook during the program and encourages adherence to activities after the conclusion may not find effect decay at a five-week interval. These adherence effects comport with the finding that motivation and effort are prerequisites for intervention efficacy (Lyubomirsky \& Layous, 2013).

Why was the effect weaker in ENHANCE-II compared to ENHANCE-I? Plausibly, the effect on positive affect, but not life satisfaction, was weaker due to the removal of two modules-gratitude and savoring - that taught participants how to extract greater hedonic well-being from the past and present. In contrast, life satisfaction may have been enhanced through the module on goals and values, which are well suited to competitive college students. Absent an intervention, their habitual focus on short-term deadlines and resume-enhancing achievements can diminish 
their intrinsic motivation (Deci et al., 1981) and distract them from allocating time to intrinsically motivated goals (Martin, 2020). Another limitation was that I did not send participants reminders to use their new knowledge, an omission that could underlie the lack of sustained gains across all outcomes.

Unlike ENHANCE-I, ENHANCE-II only used printed material. Neither lectures nor online content were used to deliver information, and any institution with a printer or photocopier can replicate this intervention. In contrast, web-based interventions present some challenges to researchers who move from one institution to another, because they may need to change the web host (K. Kushlev, personal communication, July 3, 2019). Furthermore, any particular web-based technology can also become obsolete, demanding additional work from web administrators (Fiadotau, 2020). On the other hand, print workbooks are easily editable if they are in rich text format and they may stimulate more engagement. Administrators can therefore customize them for any population. Furthermore, participants who prefer to use a computer can be given the electronic file rather than the print version. One putative benefit of online interventions is that they are self-managed (Bolier \& Abello, 2014), but paper-and-pencil interventions are also self-managed. Lastly, privacy laws inhibit the use of online interventions but not paper-and-pencil interventions in European countries (Andersson \& Titov, 2014).

\subsection{Limitations and Future Directions}

The timing of this study is a liability - the COVID-19 pandemic impinged upon all participants, and the compressed semester with earlier final exams may have put more stress on participants at the follow-up measurement. Participants were not asked to report whether they faced obstacles in attempting some activities, making it impossible to know how many assigned activities were infeasible due to shutdowns. However, global tragedies can cause people to make downward comparisons if they are unscathed by the stressor (Heim et al., 2004), so it is uncertain whether effects were accentuated, diminished, or unaffected by the pandemic. This study also did not measure well-being at distal follow-ups, and thus long-term effects cannot be ascertained. Given more funding, a study with more follow-ups and compensation for responses at each follow-up would be productive.

Because the study was conducted at a highly competitive university, the participants were likely higher than average in conscientiousness and intelligence. Students with these traits excel in high school, which increases their likelihood of being accepted at this university (Dumfart \& Neubauer, 2016; Heaven \& Ciarrochi, 2008; Ivcevic \& Brackett, 2014; Laidra et al., 2007; Zuffianò et al., 2013). Participants may have therefore been more diligent and efficient at completing exercises than the average person, which limits the generalizability of this study. The results may mainly generalize to people enrolled in competitive universities or employed in certain professional fields, where intelligence and conscientious are selected for.

The absence of a control group may also strike some readers as a limitation. However, given the well-documented stability of life satisfaction (Steger \& Kashdan, 2007), it is unlikely that increases in life satisfaction occurred due to chance, especially 
among college students who experience greater stress as a semester progresses. It is noteworthy that the average participant had greater life satisfaction at the posttest, and high-adherence participants had greater life satisfaction at the follow-up.

A recent meta-analysis of multi-component positive psychology interventions used six criteria for assessing the risk of bias (Hendriks et al., 2020; see also Higgins et al., 2011), The first two criteria, randomization and allocation concealment, are moot for this study because there is no control condition. The other four criteria were met: (a) outcome measures were administered via online questionnaires, (b) there was a description of participant dropout in the manuscript, (c) a power analysis was conducted, and (d) an intention-to-treat analysis was conducted (i.e., non-adhering participants were retained).

\section{Conclusion}

As positive psychology interventions continue to proliferate, applied researchers will be challenged to select the best interventions and place them in a meaningful sequence. ENHANCE-I and ENHANCE-II are both projects in this vein. The current study may move positive psychology one step closer to the goal of optimal sequencing by shedding light on the costs and benefits of abridgement. The tested intervention had some loss of efficacy but still elicited the desired effect. For people who are too busy to engage in a long program, an effect of this magnitude may be enough.

\section{Appendix}

\section{Message Sent to Disqualified Participants with High Modified PHQ-9 Scores}

Dear

Thanks again for filling the screener for the happiness study. One of the reasons we used the screener was to exclude people who have severe depression. Your answers to the questions to the screener suggest that you have severe depression, although only a licensed psychotherapist can truly diagnose this.

We're trying to replicate a previous study that also excluded people with severe depression. We therefore can't include you in the research portion of the study-we can't ask you to fill out questionnaires and thus we can't give you a gift card for filling them out. You can still do the happiness exercises, however. When the study workbook is ready, if you would like, you can get the workbook and do the exercises on your own until you finish them. Let me know if you would like to do that.

Since your score was high, you may also want to consider individual or group counseling. You can reach the Georgia Tech counseling center at [REDACTED] during business hours. If you call after $5 \mathrm{pm}$, select option 1 to speak with the 
after-hours counselor, and include your full name, phone number, and your school ID. You could also visit your doctor to ask for help in finding a therapist near you.

Please note that when looking for appropriate treatment options, you may want to consider the following:

-Look for a psychological or psychiatric treatment with scientific research supporting its effectiveness.

-Find a treatment provider with whom you feel comfortable. This means it is absolutely within your rights to ask questions before committing to any specific type of treatment, whether it be psychotherapy or medication.

Authors' contributions The sole author was responsible for all aspects of this research.

Funding The research was funded by SPSP Small Research Grant No. 20-1-005 and the investigator was supported by the National Science Foundation under Grant No. 1730262.

Availability of data and material The data and materials are available at https://osf.io/4tv5n

\section{Declarations}

Conflicts of interest The author declares that he has no conflict of interest.

Ethics approval The research method was consistent with ethical standards and declared exempt from review by the Institutional Review Board at Georgia Institute of Technology, Atlanta, GA.

Consent to participate All participants granted informed consent before participation.

\section{References}

Algoe, S. B., Fredrickson, B. L., \& Gable, S. L. (2013). The social functions of the emotion of gratitude via expression. Emotion, 13(4), 605-609. https://doi.org/10.1037/a0032701

Andersson, G., \& Titov, N. (2014). Advantages and limitations of Internet-based interventions for common mental disorders. World Psychiatry, 13(1), 4-11. https://doi.org/10.1002/wps.20083

Armenta, C., Bao, K. J., Lyubomirsky, S., \& Sheldon, K. M. (2014). Is lasting change possible? Lessons from the Hedonic Adaptation Prevention model. In R. E. Lucas (Ed.), Stability of Happiness (pp. 57-74). Elsevier. https://doi.org/10.1016/B978-0-12-411478-4.00004-7

Baghurst, T., \& Kelley, B. C. (2014). An Examination of Stress in College Students Over the Course of a Semester. Health Promotion Practice, 15(3), 438-447. https://doi.org/10.1177/1524839913510316

Bolier, L., \& Abello, K. M. (2014). Online positive psychological interventions: State of the art and future directions. In A. Parks \& S. M. Schueller (Eds.), Wiley-Blackwell handbook of positive psychological interventions (pp. 286-309). Wiley-Blackwell.

Buschor, C., Proyer, R. T., \& Ruch, W. (2013). Self- and peer-rated character strengths: How do they relate to satisfaction with life and orientations to happiness? The Journal of Positive Psychology, 8(2), 116-127. https://doi.org/10.1080/17439760.2012.758305 
Butler, A., Chapman, J., Forman, E., \& Beck, A. (2006). The empirical status of cognitive-behavioral therapy: A review of meta-analyses. Clinical Psychology Review, 26(1), 17-31. https://doi.org/10. 1016/j.cpr.2005.07.003

Campbell, C. M., Dortch, D., \& Burt, B. A. (2018). Reframing rigor: A modern look at challenge and support in higher education. New Directions for Higher Education, 2018(181), 11-23.

Chi, M. T. H., \& Wylie, R. (2014). The ICAP framework: Linking cognitive engagement to active learning outcomes. Educational Psychologist, 49(4), 219-243. https://doi.org/10.1080/00461520.2014. 965823

Daly, M., \& Robinson, E. (2020). Psychological distress and adaptation to the COVID-19 crisis in the United States. Journal of Psychiatric Research. https://doi.org/10.1016/j.jpsychires.2020.10.035

De Neve, J.-E., \& Oswald, A. J. (2012). Estimating the influence of life satisfaction and positive affect on later income using sibling fixed effects. Proceedings of the National Academy of Sciences, 109(49), 19953-19958. https://doi.org/10.1073/pnas.1211437109

Deci, E. L., Betley, G., Kahle, J., Abrams, L., \& Porac, J. (1981). When Trying to Win. Personality and Social Psychology Bulletin, 7(1), 79-83. https://doi.org/10.1177/014616728171012

Deci, E. L., Koestner, R., \& Ryan, R. M. (1999). A meta-analytic review of experiments examining the effects of extrinsic rewards on intrinsic motivation. Psychological Bulletin, 125(6), 627-668. https:// doi.org/10.1037/0033-2909.125.6.627

Deci, E. L., \& Ryan, R. M. (1985). Intrinsic Motivation and Self-Determination in Human Behavior. Kluwer Academic.

Diener, E., Emmons, R. A., Larsen, R. J., \& Griffin, S. (1985). The satisfaction with life scale. Journal of Personality Assessment, 49(1), 71-75. https://doi.org/10.1207/s15327752jpa4901_13

Diener, E., Inglehart, R., \& Tay, L. (2013). Theory and validity of life satisfaction scales. Social Indicators Research, 112(3), 497-527. https://doi.org/10.1007/s11205-012-0076-y

Diener, E., Lucas, R. E., \& Oishi, S. (2018). Advances and Open Questions in the Science of Subjective Well-Being. Collabra Psychology, 4(1), 15. https://doi.org/10.1525/collabra.115

Diener, E., Pressman, S. D., Hunter, J., \& Delgadillo-Chase, D. (2017). If, why, and when subjective well-being influences health, and future needed research. Applied Psychology: Health and WellBeing, 9(2), 133-167. https://doi.org/10.1111/aphw.12090

Diener, E., Sandvik, E., \& Pavot, W. (1991). Happiness is the frequency, not the intensity, of positive versus negative affect. In F. Strack, M. Argyle, \& N. Schwarz (Eds.), Subjective well-being: An interdisciplinary perspective (pp. 119-139). Pergamon.

Diener, E., Wirtz, D., Tov, W., Kim-Prieto, C., Choi, D., Oishi, S., \& Biswas-Diener, R. (2010). New well-being measures: Short scales to assess flourishing and positive and negative feelings. Social Indicators Research, 97(2), 143-156. https://doi.org/10.1007/s11205-009-9493-y

Dumfart, B., \& Neubauer, A. C. (2016). Conscientiousness Is the Most Powerful Noncognitive Predictor of School Achievement in Adolescents. Journal of Individual Differences, 37(1), 8-15. https://doi. org/10.1027/1614-0001/a000182

Dunn, E. W., Aknin, L. B., \& Norton, M. I. (2014). Prosocial Spending and Happiness. Current Directions in Psychological Science, 23(1), 41-47. https://doi.org/10.1177/0963721413512503

Faul, F., Erdfelder, E., Lang, A.-G., \& Buchner, A. (2007). G*Power 3: A flexible statistical power analysis program for the social, behavioral, and biomedical sciences. Behavior Research Methods, 39(2), 175-191.

Fava, G. A. (1999). Well-Being therapy: Conceptual and technical issues. Psychotherapy and Psychosomatics, 68(4), 171-179. https://doi.org/10.1159/000012329

Fiadotau, M. (2020). Growing old on Newgrounds: The hopes and quandaries of Flash game preservation. First Monday. https://doi.org/10.5210/fm.v25i8.10306

Forest, J., Mageau, G. A., Crevier-Braud, L., Bergeron, É., Dubreuil, P., \& Lavigne, G. L. (2012). Harmonious passion as an explanation of the relation between signature strengths' use and well-being at work: Test of an intervention program. Human Relations, 65(9), 1233-1252. https://doi.org/10. $1177 / 0018726711433134$

Gottman, J. M., \& Levenson, R. W. (1992). Marital processes predictive of later dissolution: Behavior, physiology, and health. Journal of Personality and Social Psychology, 63(2), 221-233. https://doi. org/10.1037/0022-3514.63.2.221

Heaven, P. C. L., \& Ciarrochi, J. (2008). Parental Styles, Conscientiousness, and Academic Performance in High School: A Three-Wave Longitudinal Study. Personality and Social Psychology Bulletin, 34(4), 451-461. https://doi.org/10.1177/0146167207311909 
Heim, C., Bierl, C., Nisenbaum, R., Wagner, D., \& Reeves, W. C. (2004). Regional prevalence of fatiguing illnesses in the United States before and after the terrorist attacks of September 11, 2001. Psychosomatic Medicine, 66(5), 672-678. https://doi.org/10.1097/01.psy.0000138116.12495.a2

Heintzelman, S. J., \& Kushlev, K. (2020). Emphasizing scientific rigor in the development, testing, and implementation of positive psychological interventions. The Journal of Positive Psychology, 15(5), 685-690. https://doi.org/10.1080/17439760.2020.1789701

Heintzelman, S. J., Kushlev, K., Lutes, L. D., Wirtz, D., Kanippayoor, J. M., Leitner, D., Oishi, S., \& Diener, E. (2020). ENHANCE: Evidence for the efficacy of a comprehensive intervention program to promote subjective well-being. Journal of Experimental Psychology: Applied, 26(2), 360-383. https://doi.org/10.1037/xap0000254

Hendriks, T., Schotanus-Dijkstra, M., Hassankhan, A., de Jong, J., \& Bohlmeijer, E. (2020). The efficacy of multi-component positive psychology interventions: A systematic review and meta-analysis of randomized controlled trials. Journal of Happiness Studies, 21(1), 357-390. https://doi.org/10. 1007/s10902-019-00082-1

Higgins, J. P. T., Altman, D. G., Gøtzsche, P. C., Jüni, P., Moher, D., Oxman, A. D., Savović, J., Schulz, K. F., Weeks, L., \& Sterne, J. A. C. (2011). The Cochrane Collaboration's tool for assessing risk of bias in randomised trials. BMJ (online), 343(7829), 1-9. https://doi.org/10.1136/bmj.d5928

Howell, A. J. (2017). Self-Affirmation Theory and the Science of Well-Being. Journal of Happiness Studies, 18(1), 293-311. https://doi.org/10.1007/s10902-016-9713-5

Ivcevic, Z., \& Brackett, M. (2014). Predicting school success: Comparing Conscientiousness, Grit, and Emotion Regulation Ability. Journal of Research in Personality, 52, 29-36. https://doi.org/10. 1016/j.jrp.2014.06.005

Khoury, B., Sharma, M., Rush, S. E., \& Fournier, C. (2015). Mindfulness-based stress reduction for healthy individuals: A meta-analysis. Journal of Psychosomatic Research, 78(6), 519-528. https:// doi.org/10.1016/j.jpsychores.2015.03.009

King, L. A. (2001). The health benefits of writing about life goals. Personality and Social Psychology Bulletin, 27(7), 798-807. https://doi.org/10.1177/0146167201277003

Kroenke, K., Spitzer, R. L., \& Williams, J. B. W. (2001). The PHQ-9: Validity of a brief depression severity measure. Journal of General Internal Medicine, 16(9), 606-613. https://doi.org/10.1046/j. 1525-1497.2001.016009606.x

Kushlev, K., Heintzelman, S. J., Lutes, L. D., Wirtz, D., Oishi, S., \& Diener, E. (2017). ENHANCE: Design and rationale of a randomized controlled trial for promoting enduring happiness \& wellbeing. Contemporary Clinical Trials, 52, 62-74. https://doi.org/10.1016/j.cct.2016.11.003

Laidra, K., Pullmann, H., \& Allik, J. (2007). Personality and intelligence as predictors of academic achievement: A cross-sectional study from elementary to secondary school. Personality and Individual Differences, 42(3), 441-451. https://doi.org/10.1016/j.paid.2006.08.001

Lanham, R. A. (1992). Revising prose. Macmillan.

Layous, K., Lee, H., Choi, I., \& Lyubomirsky, S. (2013). Culture Matters When Designing a Successful Happiness-Increasing Activity. Journal of Cross-Cultural Psychology, 44(8), 1294-1303. https:// doi.org/10.1177/0022022113487591

Lipsey, M. W., \& Wilson, D. B. (1993). The efficacy of psychological, educational, and behavioral treatment: Confirmation from meta-analysis. American Psychologist, 48(12), 1181-1209. https://doi.org/ 10.1037/0003-066X.48.12.1181

Lyubomirsky, S., Dickerhoof, R., Boehm, J. K., \& Sheldon, K. M. (2011). Becoming happier takes both a will and a proper way: An experimental longitudinal intervention to boost well-being. Emotion, 11(2), 391-402. https://doi.org/10.1037/a0022575

Lyubomirsky, S., King, L., \& Diener, E. (2005a). The Benefits of Frequent Positive Affect: Does Happiness Lead to Success? Psychological Bulletin, 131, 803-855. https://doi.org/10.1037/0033-2909. 131.6.803

Lyubomirsky, S., \& Layous, K. (2013). How do simple positive activities increase well-being? Current Directions in Psychological Science, 22(1), 57-62. https://doi.org/10.1177/0963721412469809

Lyubomirsky, S., Sheldon, K. M., \& Schkade, D. (2005b). Pursuing happiness: The architecture of sustainable change. Review of General Psychology, 9(2), 111-131. https://doi.org/10.1037/10892680.9.2.111

Martin, C. C. (2020). Why do college counselors perceive anxiety as increasing? A semi-structured examination of five causes. Journal of College Student Psychotherapy, . https://doi.org/10.1080/ 87568225.2020 .1753611 
Neff, K. D., Kirkpatrick, K. L., \& Rude, S. S. (2007). Self-compassion and adaptive psychological functioning. Journal of Research in Personality, 41(1), 139-154. https://doi.org/10.1016/j.jrp. 2006.03.004

Nezlek, J. B. (2011). Multilevel Modeling for Social and Personality Psychology. SAGE Publications.

Nezlek, J. B. (2017). A practical guide to understanding reliability in studies of within-person variability. Journal of Research in Personality, 69, 149-155. https://doi.org/10.1016/j.jrp.2016.06.020

Parks, A. C., \& Schueller, S. M. (2014). The Wiley Blackwell Handbook of Positive Psychological Interventions. John Wiley \& Sons, Ltd. https://doi.org/10.1002/9781118315927

Peterson, S. J., Luthans, F., Avolio, B. J., Walumbwa, F. O., \& Zhang, Z. (2011). Psychological capital and employee performance: A latent growth modeling approach. Personnel Psychology, 64(2), 427-450. https://doi.org/10.1111/j.1744-6570.2011.01215.x

Pitt, A., Oprescu, F., Tapia, G., \& Gray, M. (2018). An exploratory study of students' weekly stress levels and sources of stress during the semester. Active Learning in Higher Education, 19(1), 61-75. https://doi.org/10.1177/1469787417731194

Polavieja, J. G. (2015). Capturing culture: A new method to estimate exogenous cultural effects using migrant populations. American Sociological Review, 80(1), 166-191. https://doi.org/10.1177/ 0003122414562600

Proyer, R. T., Wellenzohn, S., Gander, F., \& Ruch, W. (2015). Toward a better understanding of what makes positive psychology interventions work: Predicting happiness and depression from the person $\times$ intervention fit in a follow-up after 3.5 years. Applied Psychology: Health and WellBeing, 7(1), 108-128. https://doi.org/10.1111/aphw.12039

Robinson, E., \& Daly, M. (2020). Explaining the rise and fall of psychological distress during the COVID-19 crisis in the United States: Longitudinal evidence from the Understanding America Study. British Journal of Health Psychology. https://doi.org/10.1111/bjhp.12493

Rohrer, J. M., \& Lucas, R. E. (2020). Causal effects of well-being on health: It's complicated. Available via PsyArxiv. https://psyarxiv.com/wgbe4. Accessed 10 Mar 2021.

Sandstrom, G. M., \& Dunn, E. W. (2014a). Is efficiency overrated? Social Psychological and Personality Science, 5(4), 437-442. https://doi.org/10.1177/1948550613502990

Sandstrom, G. M., \& Dunn, E. W. (2014b). Social Interactions and Well-Being. Personality and Social Psychology Bulletin, 40(7), 910-922. https://doi.org/10.1177/0146167214529799

Shin, L. J., Armenta, C. N., Kamble, S. V., Chang, S.-L., Wu, H.-Y., \& Lyubomirsky, S. (2020). Gratitude in collectivist and individualist cultures. The Journal of Positive Psychology, 15(5), 598604. https://doi.org/10.1080/17439760.2020.1789699

Silva, A. J., \& Caetano, A. (2013). Validation of the Flourishing Scale and Scale of Positive and Negative Experience in Portugal. Social Indicators Research, 110(2), 469-478. https://doi.org/ 10.1007/s11205-011-9938-y

Sin, N. L., \& Lyubomirsky, S. (2009). Enhancing well-being and alleviating depressive symptoms with positive psychology interventions: A practice-friendly meta-analysis. Journal of Clinical Psychology, 65(5), 467-487. https://doi.org/10.1002/jclp.20593

Steger, M. F., \& Kashdan, T. B. (2007). Stability and specificity of meaning in life and life satisfaction over one year. Journal of Happiness Studies, 8(2), 161-179. https://doi.org/10.1007/ s10902-006-9011-8

Stiwne, E. E., \& Jungert, T. (2010). Engineering students' experiences of transition from study to work. Journal of Education and Work, 23(5), 417-437. https://doi.org/10.1080/13639080.2010. 515967

Sumi, K. (2014). Reliability and validity of Japanese versions of the Flourishing Scale and the Scale of Positive and Negative Experience. Social Indicators Research, 118(2), 601-615. https://doi. org/10.1007/s11205-013-0432-6

Taaffe Young, K., Davis, K., Schoen, C., \& Parker, S. (1998). Listening to Parents. Archives of Pediatrics \& Adolescent Medicine. https://doi.org/10.1001/archpedi.152.3.255

Times Higher Education. (2021). The World University Rankings: Georgia Institute of Technology. https://www.timeshighereducation.com/world-university-rankings/georgia-institute-technology. Accessed 15 Jan 2021.

U.S. News \& World Report. (2021). Georgia Institute of Technology. https://www.usnews.com/best-colle ges/georgia-institute-oftechnology-1569/overall-rankings. Accessed 15 Jan 2021.

Watson, D., Clark, L. A., \& Tellegen, A. (1988). Development and validation of brief measures of positive and negative affect: The PANAS scales. Journal of Personality and Social Psychology, 54, 1063-1070. 
Wittenberg, B. M., Beverung, L., Ansari, A., Jacobvitz, D., \& Hazen, N. (2017). Gender differences in parents' prenatal wishes for their children's future: A mixed-methods study. Journal of Child and Family Studies, 26(7), 1865-1874. https://doi.org/10.1007/s10826-017-0713-9

Zuffianò, A., Alessandri, G., Gerbino, M., Luengo Kanacri, B. P., Di Giunta, L., Milioni, M., \& Caprara, G. V. (2013). Academic achievement: The unique contribution of self-efficacy beliefs in selfregulated learning beyond intelligence, personality traits, and self-esteem. Learning and Individual Differences, 23, 158-162. https://doi.org/10.1016/j.lindif.2012.07.010

Publisher's Note Springer Nature remains neutral with regard to jurisdictional claims in published maps and institutional affiliations. 